\title{
LA ALTERNANCIA PREDICATIVA EN ESPAÑOL
}

\author{
DOLORES AGENJO RECUERO \\ Universidad Carlos III de Madrid \\ doloresagenjo@outlook.es \\ ORCID: 0000-0001-8208-3783
}

\section{RESUMEN}

En español, la alternancia predicativa (tener granos en la cara / tener la cara con granos) difiere de la alternancia locativa (cargar el heno en el camión / cargar el camión con heno) en tres aspectos relevantes: (i) argumentos verbales, (ii) estructura eventiva y (iii) relación semántica entre el significado verbal de la variante de locado y el significado verbal en la variante de locación. Por otra parte, el tipo de construcciones propio de la alternancia predicativa no solo se combina con verbos estativos, sino también con verbos aspectualmente dinámicos, incluidos los más representativos de la alternancia locativa. En conclusión, sostenemos que la alternancia predicativa constituye un tipo de diátesis verbal distinto e independiente de la alternancia locativa.

PALABRAS CLAVE: alternancia locativa, alternancia predicativa, verbos estativos transitivos, verbos de desplazamiento y resultado, verbos de manera de movimiento, estructura eventiva.

\section{PREDICATIVE ALTERNATION IN SPANISH}

\section{ABSTRACT}

In Spanish, predicative alternation (tener granos en la cara 'have pimples on the face' / tener la cara con granos 'have a face with pimples') differs from locative alternation (cargar el heno en el camión 'load the hay into the truck' / cargar el camión con heno 'load the truck with hay') in three relevant aspects: (i) verbal arguments, (ii) event structure, and (iii) semantic relationship between verbal meaning of locatum variant and verbal meaning of location variant. On the other hand, predicative alternation constructions not only can appear with stative verbs, but also with several aspectually dynamic verbs, including those more typical in locative alternation. Then, we conclude that predicative alternation constitutes a different and independent type of verbal diathesis from locative alternation.

KEYWORDS: locative alternation, predicative alternation, stative transitive verbs, path and result verbs, manner of motion verbs, event structure.

\section{INTRODUCCIÓN}

La finalidad de este trabajo es mostrar las diferencias estructurales y semánticas entre la alternancia locativa en español (AL) y un tipo de alternancia, denominada por Cifuentes (2008) alternancia predicativa. Con tal propósito, nos centraremos en el análisis y comparación de tres aspectos clave para ambos tipos de alternancia: argumentos verbales, estructura eventiva y relación 
semántica entre el significado verbal de la variante de locado y el significado verbal en la variante de locación. Los principios teóricos de nuestro análisis se basan en los estudios sobre la AL realizados desde la perspectiva del proyeccionismo léxico por Levin (2003, 2006), Levin y Rappaport Hovav (1998), Rappaport Hovav y Levin (1988), y, desde el proyeccionismo sintáctico, por Mateu (2000, 2002, 2017). Por lo que concierne a la estructura eventiva de los verbos implicados en las alternancias que aquí se analizan, adoptamos el modelo sintáctico-aspectual elaborado por Ramchand (2008).

\section{Argumentos Verbales}

El fenómeno representado por la AL consiste en la capacidad de ciertos verbos, a los que denominaremos verbos alternantes (VAL), para insertarse en dos construcciones sintácticas de argumentos idénticos que intercambian las posiciones sintácticas de objeto directo (OD) y oblicuo (OBL). ${ }^{1}$ Así, como puede observarse en (1)-(5), el objeto locado (cajas, espray, queso rallado, helado, trapo) es $\mathrm{OD}$ en los ejemplos (a) y (OBL) en los de (b); mientras que la ubicación (el maletero, su pelo, la sopa, la pared, el suelo) es el OBL en los ejemplos (a) y el OD en los de (b).

(1) a. Juan cargó cajas en el maletero.

b. Juan cargó el maletero con cajas.

(2) a. María roció espray en su pelo.

b. María roció su pelo con espray.

(3) a. El cocinero espolvoreó queso rallado en la sopa.

b. El cocinero espolvoreó la sopa con queso rallado.

(4) a. El niño salpicó helado en la pared.

b. El niño salpicó la pared con helado.

(5) a. Pedro frotó un trapo en el suelo.

b. Pedro frotó el suelo con un trapo.

Atendiendo exclusivamente al argumento que ocupa la posición de OD, denominaremos variante de locado al tipo de construcción ejemplificado en (1a)(5a) y variante de locación al de (1b)-(5b). ${ }^{2}$

\footnotetext{
${ }^{1}$ Con la denominación AL nos referimos exclusivamente a las alternancias de tipo transitivo. Quedan, por tanto, fuera de nuestro estudio ejemplos como los representados por el agua rebosaba en la bañera / la bañera rebosaba de agua. Para un análisis de este tipo de alternancia intransitiva, véanse Levin y Rappaport Hovav (1995) y Mayoral Hernández (2015). Cabe subrayar, por otra parte, que no todos los autores coinciden en incluir este fenómeno dentro de la AL (véase al respecto Dowty 2000).

${ }^{2}$ Los términos para referirse a las variantes de la AL son diversos. Principalmente se basan en criterios de tres tipos: formales, temáticos y aspectuales. Así, centrándose en las preposiciones
} 
Los verbos que intervienen en este tipo de construcciones se caracterizan por referirse a eventos dinámicos, ya sea de desplazamiento y resultado (VDR) - cargar, rociar, espolvorear, salpicar-, ${ }^{3}$ ya sea a eventos de manera de movimiento (VMM) - frotar, golpear, friccionar. ${ }^{4}$ Pero existe también un conjunto de verbos estativos transitivos (VET) que pueden intervenir en construcciones aparentemente similares a las de (1)-(5), como podemos apreciar en (6)-(10).

(6) a. Lucir canas en la barba es algo que da un toque de distinción. [ejemplo tomado de Patiño (2017)]

b. Lucir la barba con canas es algo que da un toque de distinción.

(7) a. Llevaba manchas de sangre en el pantalón y la camisa.

b. Llevaba el pantalón y la camisa con manchas de sangre. [ejemplo tomado de F. V. (2010)]

(8) a. Consejos para mantener buen aspecto en tu piel.

b. Consejos para mantener tu piel con buen aspecto. [ejemplo tomado de Frost (2017)]

(9) a. La mujer joven con la lupa muestra acné en la piel.

b. La mujer joven con la lupa muestra la piel con acné. [ejemplo tomado de Kotin (2017)]

(10) a. Tiene granos en la cara.

b. Tiene la cara con granos.

[ejemplos (10a-b) tomados de Cifuentes (2008: 52)]

Cifuentes (2008: 51-56) considera que los verbos del tipo ejemplificado en (6)-(10) constituyen una clase especial de AL que denomina alternancia predicativa (AP). Así, tomando como referencia los ejemplos de este autor

características de cada construcción, Iwata (2005, 2008), Pinker (1989), Rappaport Hovav y Levin (1988) utilizan los términos into/onto variant y with variant. Por su parte, Brinckman (1997) y Nichols (2008) eligen las denominaciones object-theme variant y object-goal variant, que destacan el diferente papel temático asignado al OD en cada construcción. En cuanto a la diferencia aspectual entre ambas variantes, queda reflejada por los términos change of location variant $\mathrm{y}$ change of state variant, utilizados por numerosos autores, como Levin y Rappaport Hovav (1998), Mateu (2000, 2002), Mayoral Hernández (2015) y Lewandowsky (2014).

${ }^{3}$ Utilizamos la denominación VDR para referirnos al grupo de VA que conjugan la expresión de un cambio de ubicación o desplazamiento en la variante de locado (cargar heno en el camión) con la de un cambio de estado o resultado en la variante de locación (cargar el camión con heno).

${ }^{4}$ Por lo general, en los trabajos sobre la AL se contempla como homogéneo el conjunto de verbos que intervienen en el fenómeno, de modo que no se realizan distinciones entre ellos, salvo las relacionadas con sus peculiaridades idiosincrásicas (véanse las clasificaciones de Cifuentes 2008, Levin 2006, Lewandowsky 2014, Pinker 1989, entre otros). Sin embargo, no todos los verbos que participan en este fenómeno expresan cambio de ubicación y cambio de estado; los que aquí denominamos VMM hacen referencia a eventos de movimiento sin desplazamiento. Como más adelante veremos, aunque no nos es posible profundizar aquí en la cuestión, estos verbos poseen características argumentales y estructurales diferenciadas. 
mostrados en (10a-b), podemos observar en (11) el claro paralelismo entre las construcciones de AP y de AL.

(11) a. Variante de locado:

$\begin{array}{llllll} & \mathrm{SD}(\mathrm{x})_{\text {sujeto }} & \mathrm{V} & \mathrm{SD}(\mathrm{y}) \mathrm{OD} & \mathrm{P} & \mathrm{SD}(\mathrm{z}) \text { oBL } \\ \mathrm{AP} & \text { Juan } & \text { tiene } & \text { granos } & \text { en } & \text { la cara. } \\ \mathrm{AL} & \text { Juan } & \text { cargó } & \text { heno } & \text { en } & \text { el camión. } \\ & \text { Juan } & \text { frotó } & \text { un trapo } & \text { en } & \text { el cristal. }\end{array}$

b. Variante de locación:

$\begin{array}{llllll} & \text { SD }(\mathrm{x})_{\text {sujeto }} & \mathrm{V} & \mathrm{SD}(\mathrm{z}) \mathrm{OD} & \mathrm{P} & \mathrm{SD}(\mathrm{y}) \text { OBL } \\ \mathrm{AP} & \text { Juan } & \text { tiene } & \text { la cara } & \text { con } & \text { granos. } \\ \mathrm{AL} & \text { Juan } & \text { cargó } & \text { el camión } & \text { con } & \text { heno. } \\ & \text { Juan } & \text { frotó } & \text { el cristal } & \text { con } & \text { un trapo. }\end{array}$

Sin embargo, esta similitud estructural es tan solo aparente, pues, como seguidamente trataremos de explicar, los verbos que intervienen en la $\mathrm{AL}$ poseen distinto número y tipo de argumentos, así como distintas estructuras argumentales y características semánticas.

\subsection{Argumentos verbales en la alternancia locativa}

Partimos de la hipótesis de que los VAL, ya sean VDR o VMM, son triádicos. Algunos autores (Mateu 2000, 2002; Jackendoff 1990) solo atribuyen función argumental al OBL en la variante de locado (Juan cargó el heno en el camión), pero no en la variante de locación (Juan cargó el camión con heno). Por nuestra parte, aunque no discutiremos aquí en profundidad la tesis de estos autores, nos decantamos por la explicación de Mayoral Hernández (2015: 60-62), ${ }^{5}$ quien atribuye a la preposición en cada variante la capacidad de asignar papeles temáticos a sus constituyentes. Así, observamos en (12b) que es la preposición en la que nos permite interpretar que los sacos son desplazados, mientras que la preposición con en (12c) indica que los sacos son el contenedor o locación de destino del objeto desplazado.

(12) a. Juan cargó los sacos.

b. Juan cargó los sacos en el coche.

c. Juan cargó los sacos con patatas.

No haremos referencia por el momento a las diferencias entre la estructura sintáctica de los VDR (cargar, rociar, espolvorear, etc.) y la que corresponde a los VMM (frotar, golpear, friccionar, etc.). Baste por ahora tan solo destacar el hecho de que unos y otros presentan una estructura triádica en que el agente funciona como sujeto sintáctico, mientras que locado y locación ocupan alternativamente las posiciones de especificador (OD) y complemento de $\mathrm{V}$ (OBL).

\footnotetext{
${ }^{5}$ Seguimos a Dowty (2003) en cuanto a la definición de los conceptos de argumento y adjunto.
} 
Así pues, los constituyentes argumentales de los VAL, para cada variante de la alternancia, serían los que quedan reflejados en (13), con indicación entre paréntesis de la función sintáctica correspondiente:

(13) a. Juan cargó el heno en el camión. <agente (sujeto), locado (OD), locación (OBL)>

b. Juan cargó el camión con el heno. <agente (sujeto), locación (OD), locado (OBL)>

\subsection{Argumentos verbales en la alternancia predicativa}

Siguiendo a Ramchand (2008: 115-116), consideramos que los VET solo poseen dos argumentos, que coinciden con el sujeto sintáctico y el OD, en posición de complemento de $\mathrm{V}$, respectivamente. Por lo que concierne al sintagma preposicional (SP), realizaría, según nuestra hipótesis, la función de complemento del núcleo del OD en ambas construcciones, tal como se refleja en (14)-(16).

(14) a. Juan tiene granos en la cara.

[Juan] [tener] [granos [en la cara]sp]sD (OD)

b. Juan tiene la cara con granos.

[Juan] [tener] [la cara [con granos]sP]sD (OD)

(15) a. Julio muestra heridas en el cuello. [ejemplo tomado de Diario Rotativo (2016)]

[Julio] [mostrar] [heridas [en el cuello]sp]sD (OD)

b. Julio muestra el cuello con heridas.

[Julio] [mostrar] [el cuello [con heridas]sp]sD (OD)

(16) a. María lucía una corona de flores en el cabello.

[ejemplo tomado de Plaza (2015)]

[María] [lucir] [una corona de flores [en el cabello]sp]sD (OD)

b. María lucía el cabello con una corona de flores.

[María] [lucir] [el cabello [con una corona de flores]sp]sD (OD)

Denominaremos poseedor al argumento sujeto y rema (propiedad poseída) al argumento OD (holder y rheme, respectivamente, en términos de Ramchand 2008: 115-116). ${ }^{6}$ La alternancia se produce porque tanto locado como locación pueden ocupar la posición de rema $y$, por tanto, de OD, siempre que entre ellos exista una relación de inclusión. ${ }^{7}$

Así, en (17), por ejemplo, al poseedor (Juan) le puede ser atribuida la propiedad (rema) de tener granos en la cara o de tener la cara con granos porque el

6 Sobre los argumentos de los verbos estativos, véanse, entre otros -además de Ramchand (2008) -, Dowty (1979), Levin (2007), MacDonald (2006), Maienborn (2008) y Rothmayr (2009).

${ }^{7}$ Otros VET se prestan a alternancias que difieren de las aquí descritas. Por ejemplo, verbos como amar, odiar, temer, admirar, etc. dan lugar a alternancias del tipo ejemplificado por Cifuentes (2008: 56) con admirar en Cristina su bondad / admirar a Cristina por su bondad, donde la propiedad poseída puede ocupar alternativamente la posición de OD o la posición de adjunto causal de V. 
locado (granos) está incluido en la locación (la cara), de modo que la posesión de uno implica necesariamente la posesión del otro, y viceversa. Consecuentemente, el estado predicado por el verbo (tener) alcanza a ambos objetos. ${ }^{8}$ Si no existe esta doble implicación entre posesión de locado y posesión de locación, la alternancia no resulta aceptable, tal como se evidencia en (17), pues nuestro conocimiento del mundo no nos permite establecer vinculación entre tener dinero en un banco (entidad financiera) y tener un banco. En cambio, en (18), tener dinero en una cuenta implica tener una cuenta.

(17) a. Juan tiene dinero en un banco.

b. \#Juan tiene un banco con dinero.

(18) a. Juan tiene dinero en una cuenta.

b. Juan tiene una cuenta con dinero.

Así pues, los constituyentes argumentales de los VET en AP, para cada variante de la alternancia, serían los que se muestran en (19).

(19) a. Juan tiene granos en la cara. <poseedor (sujeto), locado (OD)>

b. Juan tiene la cara con granos. $<$ poseedor (sujeto), locación (OD)>

\section{ESTRUCTURA EVENTIVA}

Además de las diferencias en cuanto a la composición argumental, los VET se diferencian de los VAL por sus características aspectuales. En este trabajo, hemos adoptado el marco teórico elaborado por Ramchand (2008) porque su modelo de estructura eventiva (EE), entendida como una estructura sintácticoaspectual, nos permite reflejar dichas diferencias aspectuales, como seguidamente pasamos a exponer.

\subsection{Verbos con alternancia locativa}

Según Ramchand (2008), la EE está formada por la composición de distintos subeventos: Subevento inicial (Sinic), Subevento de proceso (Sproc) y Subevento de Resultado (Sres). Así, los distintos tipos de eventos se caracterizarían por la distinta combinación de estos componentes, tal como se muestra en (20).

\footnotetext{
${ }^{8}$ La relación definida por los VET entre los objetos implicados en la alternancia cumpliría la propiedad transitiva: si Juan tiene una casa y la casa tiene piscina, Juan tiene piscina. A la inversa (si Juan tiene una piscina y la piscina está en la casa, Juan tiene la casa), la inferencia, obviamente, se apoyaría en nuestro conocimiento del mundo, que sanciona como normal la vinculación entre tener una piscina en una casa y tener una casa, o, por ejemplo, entre tener varices en las piernas y tener piernas.
} 
(20) a. Eventos estativos: [Sinic]

b. Eventos dinámicos atélicos: [Sinic, Sproc]

c. Eventos télicos: [Sinic, Sproc, Sres]

Así pues, siguiendo este modelo, entre los VAL cabe distinguir tres grupos aspectuales y, por tanto, tres tipos de EE: (i) verbos siempre télicos (pinchar, salpicar), que designan eventos puntuales; (ii) verbos siempre atélicos (frotar, friccionar, restregar), y (iii) verbos optativamente télicos (cargar, untar, rociar), que admiten una interpretación télica si su OD está delimitado - véase (21)-(23).

(21) a. Juan casi salpica helado en la pared.

b. Juan casi salpica la pared con helado.

(22) a. Juan frotó \{el trapo/trapos\} en el suelo \{durante media hora / *en una hora\}.

b. Juan frotó \{el suelo/suelos\} con el trapo \{durante media hora / *en una hora\}.

(23) a. Juan cargó \{el heno/heno\} en el camión \{en una hora/durante una hora\}.

b. Juan cargó \{el camión/camiones\} con heno en una hora \{en una hora/durante una hora\}.

Nuestra hipótesis es que los primeros podrían encajar en una EE similar a la que Ramchand propone para verbos télicos como poner, comprar, enviar: [Sinic, Sproc, Sres], tal como se refleja en (24).

(24) Juan salpicó helado en la pared. / Juan salpicó la pared con helado.

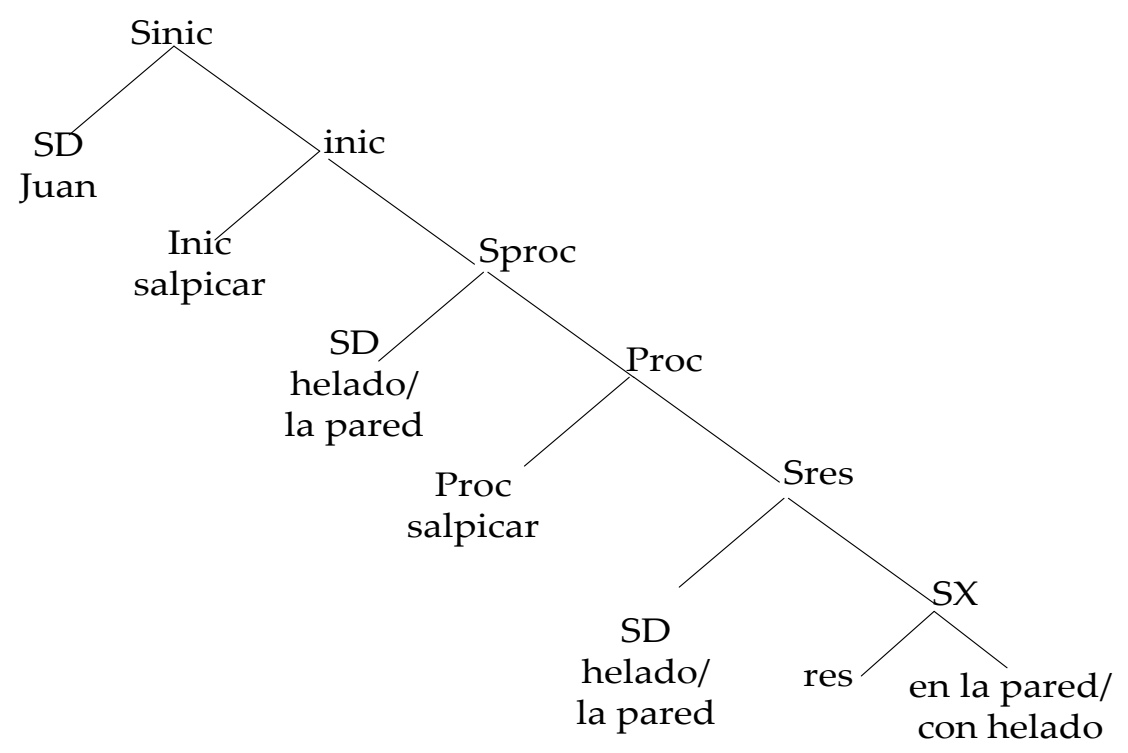

Por lo que se refiere al grupo (ii), nuestro análisis se ajusta al de Ramchand (2008: 73-74) para los verbos siempre atélicos, representados por empujar; mientras que los del grupo (iii) encajan con el que esta misma autora propone para los verbos de consunción, del tipo de comer y leer. En ambos casos, las respectivas EE carecen de Sres y su diferencia radica exclusivamente en la posición del OD: especificador de proc en los verbos siempre atélicos (frotar, 
restregar, golpear, etc.) y complemento de proc en los verbos de consunción (cargar, rociar, untar, etc.) - véanse (25) y (26), respectivamente.

(25) Juan frotó el trapo en el suelo. / Juan frotó el suelo con el trapo.

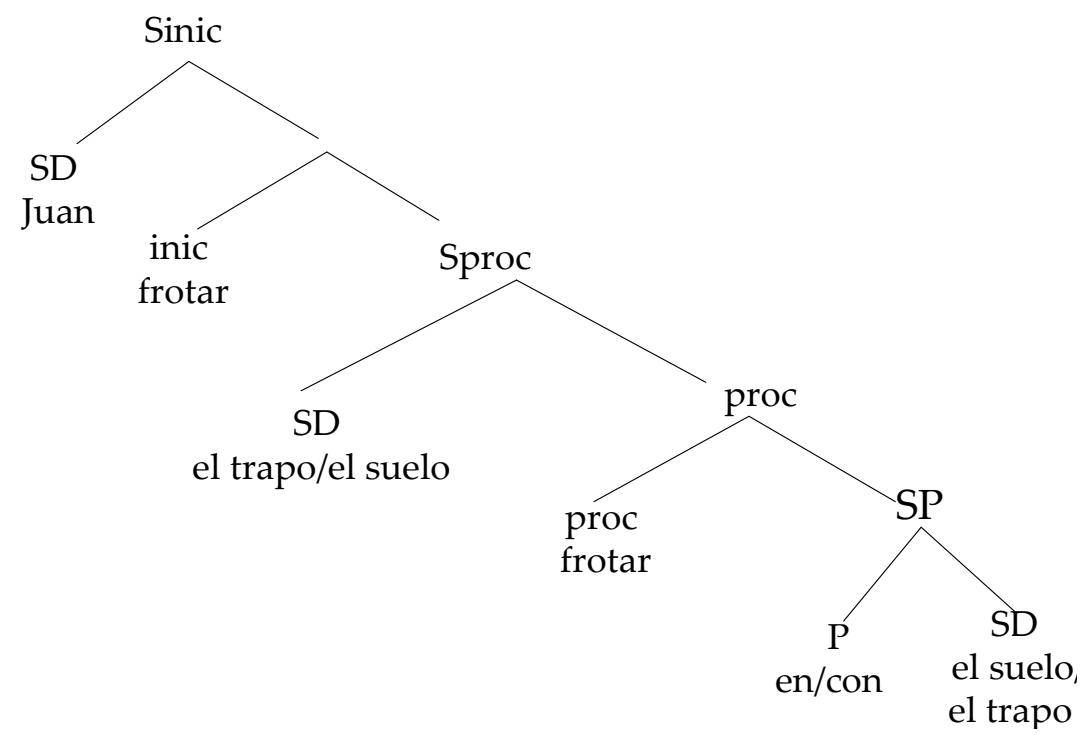

(26) Juan cargó el heno en el camión. / Juan cargó el camión con el heno.

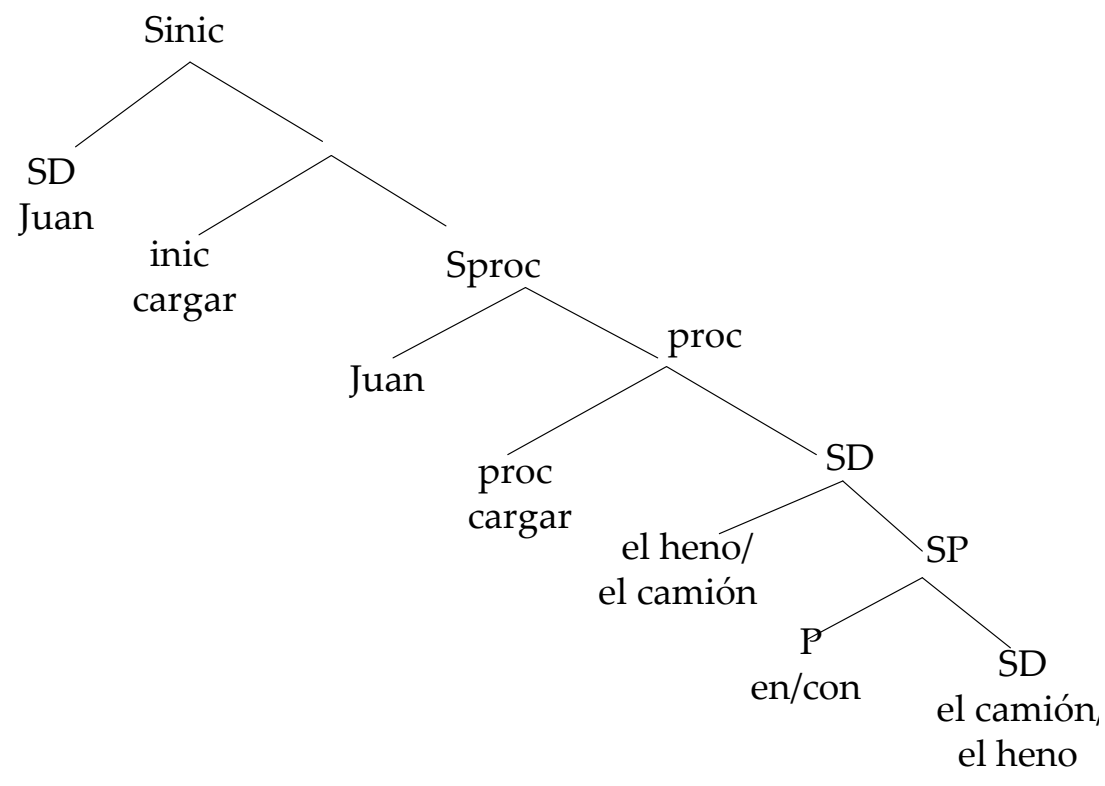

\subsection{Verbos estativos transitivos con alternancia predicativa}

Ramchand (2008: 115-116) asigna a los verbos del tipo representado por fear (estativos transitivos) un único marcador aspectual (inic) y una EE, compuesta exclusivamente por Sinic, como la ejemplificada por Katherine fears nightmares 'Katherine teme las pesadillas'. Creemos que dicha estructura se adecua perfectamente a los VET que aquí nos ocupan. En dicha EE observamos los siguientes aspectos: (i) el carácter diádico: poseedor y rema, representados por 
SD (x) y SD (y), respectivamente, son los dos argumentos verbales, y (ii) SDoD ocupa la posición de complemento de inic, tal como reflejamos en (27).

(27) Katherine fears nightmares.

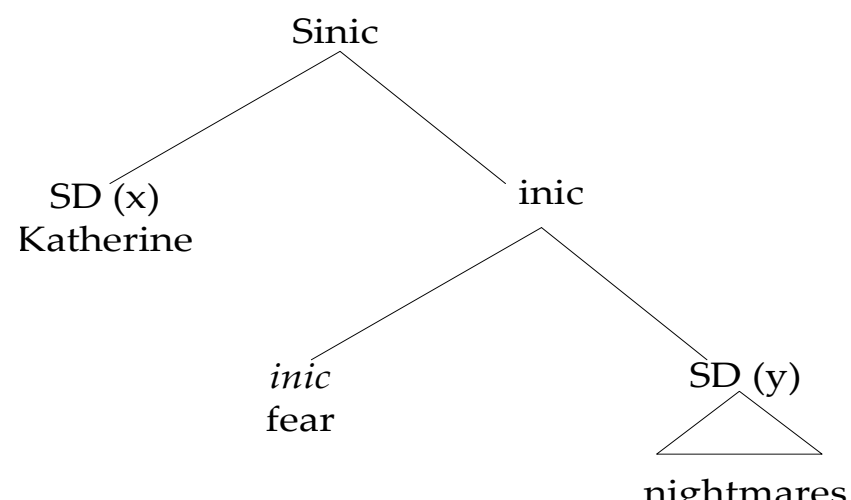

nightmares

Así pues, siguiendo el modelo aspectual propuesto por Ramchand para el verbo inglés fear y de acuerdo con la composición argumental diádica de los VET, la EE que proponemos para la alternancia predicativa de este tipo de verbos es la que se muestra ejemplificada en (28).

(28) Juan luce canas en la barba. / Juan luce la barba con canas.

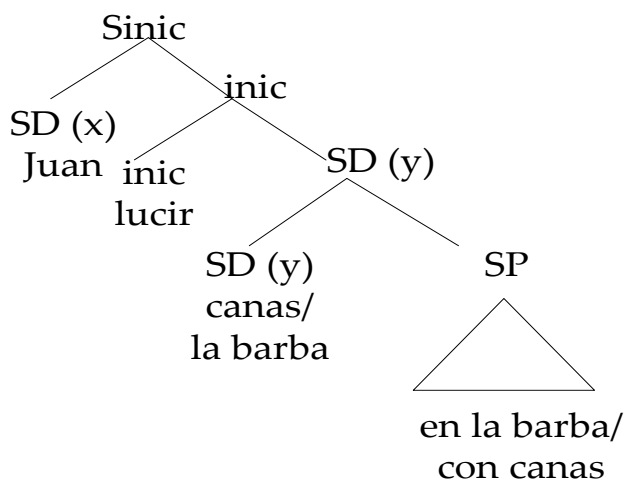

Los complementos de inic en ambas variantes de la AP (canas en la barba y barba con canas) equivalen a sendas cláusulas reducidas (small clauses), que, de acuerdo con Stowell (1981, 1983), funcionan como constituyentes oracionales - en ambos casos OD de inic (lucir)-, donde SP (en la barbalcon canas) constituye el predicado de SD (y) (canas/la barba).

Las estructura representada en (28) nos permite realizar las siguientes observaciones: (i) la ausencia de Sproc distingue aspectualmente a los VET del conjunto de verbos que intervienen en la AL; (ii) SD (x) representa al poseedor y coincide con el sujeto sintáctico de Sinic; (iii) locado y locación alternan en la posición de SDoD; (iv) en la variante de locado (lucir canas en la barba) el SP encabezado por en ocupa la posición de complemento del locado (canas), y en la variante de locación (lucir la barba con canas), el SP encabezado por con, la de complemento de la locación (barba), tal como se refleja en (29). 
(29) VET: posiciones sintácticas de locado y locación en la alternancia predicativa

\author{
Posición sintáctica \\ Inic (núcleo de Sinic) \\ SD Sujeto de inic \\ SD OD (complemento de inic) \\ SP Complemento de OD
}

Variante de locado
lucir
Juan
canas
en la barba

Variante de locación

lucir

Juan

la barba

con canas

\section{RELACIÓN SEMÁNTICA ENTRE LAS RAÍCES VERBALES DE LAS VARIANTES}

Además de las diferencias observadas en cuanto a constituyentes argumentales y EE, la relación semántica entre el significado verbal en la variante de locado y el significado verbal en la variante de locación también nos permite diferenciar entre VAL y VET.

Respecto a los primeros, compartimos el criterio según el cual las raíces verbales de las variantes no son sinónimas, aunque comportan la misma forma (véanse al respecto Rappaport Hovav y Levin 1988; Levin y Rappaport Hovav 1998; Mateu 2000, 2002, 2017; Pinker 1989; Baker 1997, entre otros muchos), sino que entre ellas se establece una oposición semántica. ${ }^{9}$ Por el contrario, en el caso de la AP, el significado verbal es idéntico en las dos variantes de la alternancia.

\subsection{Verbos con alternancia locativa}

En la AL, la diferencia de significado adquirido por la raíz verbal en una y otra variante es, a nuestro juicio, resultado de la incorporación de la preposición a la raíz verbal, tal como se muestra más abajo, en (30). Así, por lo que concierne a los VDR, la incorporación al verbo causativo $\mathrm{V}$ de la preposición de coincidencia terminal (en, sobre, por, etc. $)^{10}$ en la variante de locado, y de la preposición de coincidencia central $(c o n, d e)^{11}$ en la variante de locación crea un contraste semántico entre los significados verbales de las respectivas variantes. Dicho contraste es, a nuestro juicio similar al que existe entre parejas no homónimas como comprar/vender, dar/recibir, y en la alternancia de dativo en

${ }^{9}$ La sinonimia entre las variantes de la AL y, por consiguiente, entre las correspondientes raíces verbales era defendida por la hipótesis derivacionista, según la cual, ambas variantes derivaban de una misma estructura profunda, de manera que la variante de locación constituía la forma básica y la variante de locación se obtenía mediante transformaciones a partir de dicha estructura profunda. Véanse al respecto Hall (1965), Fillmore (1968) y Larson (1990). Sin embargo, siguiendo el criterio de Anderson (1971), la mayoría de los autores actuales desechan esta hipótesis y atribuyen un significado distinto a cada variante.

${ }^{10}$ En relación al análisis de los SP direccionales en español, véanse Bosque (1997: 146) y García Miguel (2006: 1284-86).

${ }^{11}$ Sobre la incorporación de la preposición a V, véanse Hale y Keyser (1993, 1999), Mateu (2000: 9-10) y Baker (1988), entre otros. 
inglés (Harley 2002, Ramchand 2008), de modo que los correspondientes significados verbales podrían ser parafraseado como se indica en (31). ${ }^{12}$

(30) Incorporación de P a V.

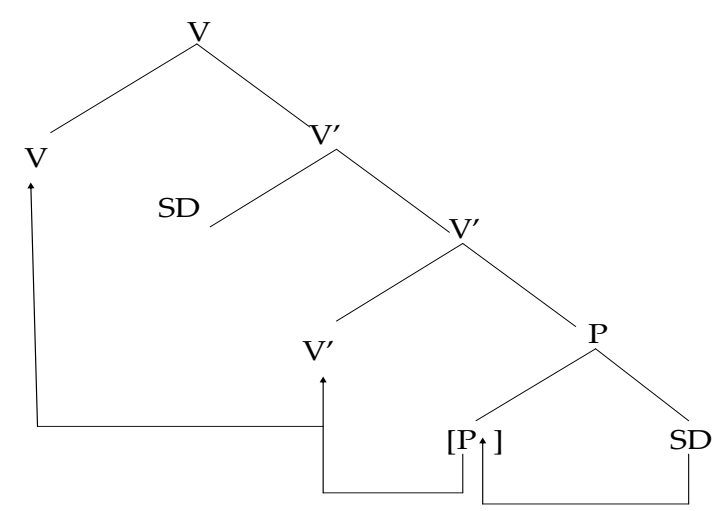

(31) a. cargar heno en el camión

[causar [ir a estar [en]]]v [heno]od [en el camión]sp

b. cargar el camión con heno

[causar [llegar a estar [con]]]v [el camión]od [con heno]sp

De igual forma, en (32) se aprecia cómo los VMM reflejan el contraste entre el significado de las raíces de una y otra variante de la alternancia: incorporación a $\mathrm{V}$ de la preposición locativa sin valor direccional (en, sobre, por, etc.) en la variante de locado, y de la preposición con, en este caso no resultativa, sino instrumental, en la variante de locación. ${ }^{13}$

(32) a. frotar el trapo en el suelo [causar [hacer frote [en]]]v [el trapo]od [en el suelo]sp

b. frotar el suelo con el trapo

[causar [hacer frote [con]]]v [el suelo]od [con el trapo]sp

\subsection{Verbos estativos transitivos en alternancia predicativa}

Aunque las variantes de la AP mantienen entre sí una relación de oposición semántica, las raíces verbales respectivas muestran idéntico significado. Nuestra hipótesis es que ello es debido a que la incidencia semántica de las preposiciones estativas (en/con) de esta alternancia se reduce al ámbito definido

${ }^{12}$ Los predicados [ir-a-estar] y [llegar-a-estar] reflejan la oposición observada por Jackendoff (1990) entre las funciones GO e INCH, que el autor asigna a las estructuras léxico conceptuales de la variante de locado y de la variante de locación, respectivamente.

${ }^{13}$ No nos detenemos aquí en explicar las diferencias entre las raíces verbales de los VDR, como cargar, y las de los VMM, como frotar. Baste decir que las características aspectuales de los segundos permiten postular a Mateu (2000), a quien seguimos en esta propuesta, una raíz abstracta ensamblada con una raíz nominal que proporciona el contenido léxico de $\mathrm{V}$ (do rub 'hacer frote'). Respecto al valor no resultativo de la preposición con en los VMM, nos basamos en la imposibilidad de su sustitución por la preposición de, característica de la AL con VDR. 
por los OD respectivos (canas en la barba / barba con canas), sin afectar en absoluto al significado de $\mathrm{V}$, es decir, sin que se produzca la incorporación de la preposición a la matriz verbal, tal como puede apreciarse en (33).

(33) a. lucir canas en la barba

[lucir]v [canas [en la barba]ss] SD (OD)

b. lucir la barba con canas

[lucir]v [la barba [con canas]ss] SD (OD)

\section{LA ALTERNANCIA PREDICATIVA EN VERBOS NO ESTATIVOS}

La AP también puede darse con otros verbos transitivos no estativos, ya sean estos alternantes o no alternantes. Por ejemplo, en el caso de un VAL, como cargar, el locado puede ser complementado tanto por un SP encabezado por en como por con - véanse (34) y (35), respectivamente.

(34) a. Pedro cargó [heno [en sacos]] en el camión.

b. Pedro cargó [sacos [con heno]] en el camión.

(35) a. Pedro cargó el camión [\{con/de\} heno [en sacos]].

b. Pedro cargó el camión [\{con/de\} sacos [\{con/de\} heno]].

Por lo que concierne a los verbos no alternantes, son numerosos los ejemplos que admiten estructuras predicativas (cláusulas reducidas) funcionando como complementos de sus SDod. Así lo evidencian verbos, tanto télicos (presentar, realizar o construir) en (36)-(38) como otros de movimiento atélico (arrastrar, empujar, llevar, etc.) en (39)-(41). ${ }^{14}$

(36) a. López presentó [más de 50 erratas y faltas de ortografía [en un escrito]].

b. López presentó [un escrito [con más de 50 erratas y faltas de ortografía]]. [ejemplo tomado de Arias (2015)]

(37) a. El alumno realizó [errores [en el ejercicio]].

b. El alumno realizó [el ejercicio [con errores]].

(38) a. Los vecinos construyeron [una piscina [en la casa]].

b. Los vecinos construyeron [una casa [con piscina]].

(39) a. El campesino arrastró [las patatas [en un saco]].

b. El campesino arrastró [un saco [con patatas]].

(40) a. María empujó [al niño [en el carrito]].

b. María empujó [el carrito [con el niño]].

\footnotetext{
${ }^{14}$ Los verbos de desplazamiento (no télicos) pueden ser complementados por un SP que los delimite (arrastrar al niño en el sofá hasta el comedor / arrastrar el sofá con el niño hasta el comedor). Estos complementos que designan la localización final de la trayectoria no pueden ocupar la posición del OD (arrastrar la habitación con el sofá).
} 
(41) a. Juan llevó [los libros [en cajas]].

b. Juan llevó [las cajas [con libros]].

Las alternancias ejemplificadas en (34)-(41), como en el caso de los VET, no afectan a $\mathrm{V}$, cuyo significado se mantiene invariable en ambas construcciones, y son posibles por la relación de pertenencia o inclusión existente entre el referente del OD y el del SP que lo complementa. Cuando tal relación no existe, no se produce alternancia alguna. Así sucede, por ejemplo, en (42) y (43), donde los SP no forman parte del SDod, sino que son adjuntos verbales que delimitan, respectivamente, la trayectoria verbal (a la habitación) y el lugar donde se produce la acción (en la calle).

(42) a. María arrastró el sofá a la habitación.

b. *María arrastró la habitación con el sofá.

(43) a. María empujó el cochecito en la calle.

b. *María empujó la calle con el cochecito.

En cuanto a los verbos télicos, ejemplificados en (37) y (38), conviene distinguirlos de VAL como grabar o tatuar. La alternancia carece de incidencia en el significado verbal de verbos del tipo de realizar o construir, pues presentan idéntico significado en ambas variantes, tal como se muestra en (44). En cambio, grabar - véase (45) - mantiene entre los significados que adquiere en una y otra construcción una relación semántica de oposición.

(44) a. Juan construyó una piscina en la casa.

[causar-estar construida]v [una piscina [en la casa]sp]od

b. Juan construyó una casa con piscina.

[causar-estar construida]v [una casa [con piscina]ss] $\mathrm{OD}$

(45) a. El orfebre grabó la fecha en la pulsera.

[causar-ir-a-estar grabada [en]]v [la fecha]od [en el reloj]sp

b. El orfebre grabó la pulsera con la fecha.

[causar-llegar-a-estar grabada [con]]v [la pulsera]od [con la fecha]sp

Estos datos nos permiten realizar las siguientes consideraciones: (i) las construcciones de AP pueden complementar al OD de verbos diversos; basta que entre el referente del OD y el del SP que lo complementa se perciba una relación de inclusión (sacos/heno, escrito/erratas, ejercicio/errores, casa/piscina, saco/patatas, carrito/niño, cajas/libros, etc.); (ii) un verbo puede combinarse con construcciones de AP (granos en la cara / cara con granos, patatas en el saco / saco con patatas, etc.) si el estado o la acción que denota alcanza a locación y locado (tener puede predicarse con idéntico significado de cara y de granos; arrastrar, de saco y patatas, etc.); (iii) la construcción de AP se circunscribe al ámbito definido por el OD, sin afectar en modo alguno al significado verbal, es decir, sin que se produzca incorporación de la preposición en la matriz verbal, tal como se refleja 
en las representaciones de la EE de arrastrar y de realizar en (46) y (47), respectivamente.

(46) María arrastró \{patatas en un saco / un saco con patatas\} hasta el almacén.

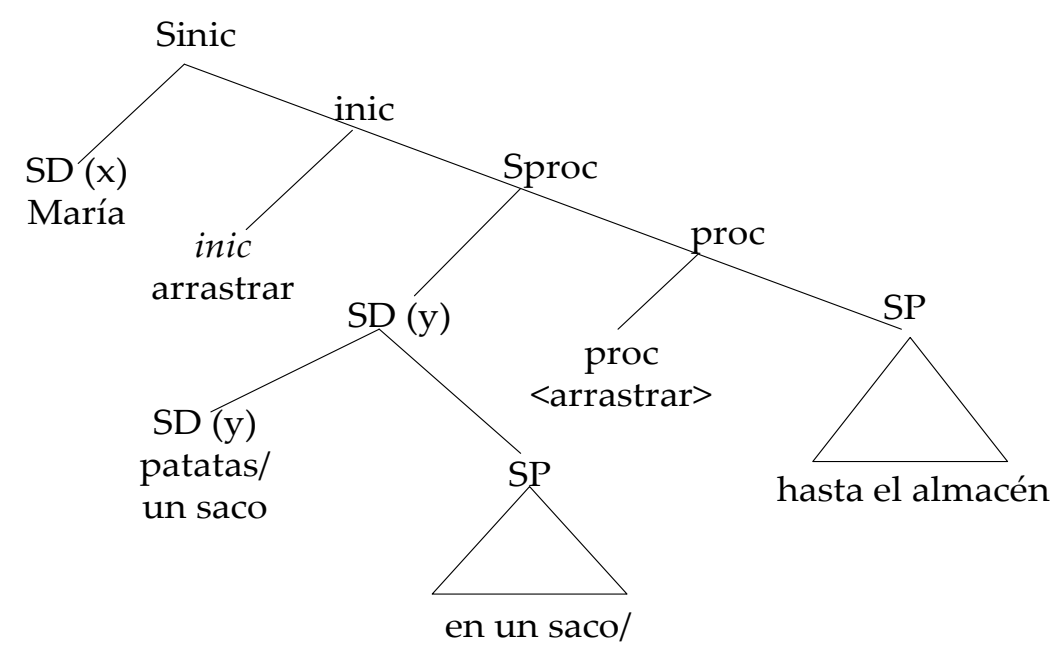

(47) María realizó \{errores en el ejercicio / el ejercicio con errores\}.

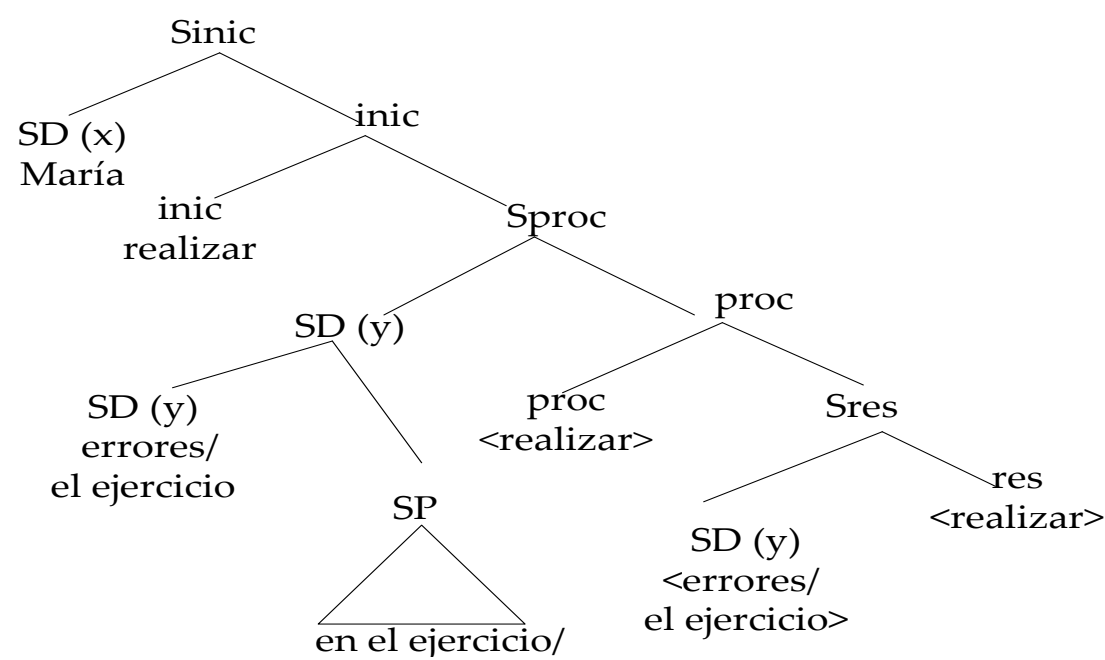

Por último, cabe subrayar, además, que posiblemente cuanto menos específico es el significado de un verbo más posibilidades tiene este de que la acción o estado denotado alcance tanto a su OD como al complemento de este. Así, mientras hacer admite las dos construcciones alternantes de (48), cometer y acabar solo se combinan con una de ellas, como muestran los ejemplos de (49) y (50), respectivamente. ${ }^{15}$

\footnotetext{
${ }^{15}$ Podríamos decir que los verbos télicos que implican acciones puntuales, como cometer, solo pueden tomar por OD al locado, mientras que los que indican finalización de un acto que comporta duración (acabar) solo pueden tener como OD a la locación. En cambio, hacer denota acción acabada, pero, en nuestra opinión, es neutro respecto a los conceptos de inicio y finalización, lo que le permite admitir ambos tipos de objetos.
} 
(48) a. Juan hizo faltas en el dictado.

b. Juan hizo el dictado con faltas.

(49) a. Juan cometió faltas en el dictado.

b. *Juan cometió el dictado con faltas.

(50) a. *Juan acabó faltas en el dictado.

b. Juan acabó el dictado con faltas.

Precisamente, el significado poco específico de los verbos estativos del tipo que aquí hemos examinado (tener, llevar, mostrar, etc.), que se limitan a atribuir una propiedad o a funcionar como cópula entre el poseedor y su propiedad, los capacita, a nuestro entender, para intervenir en este tipo de alternancias.

\section{CONCLUSIONES}

Como resultado de nuestro análisis, podemos establecer las siguientes conclusiones:

a) Los VAL reúnen las siguientes propiedades (i) tienen estructura argumental triádica, (ii) constituyen siempre eventos dinámicos, por lo que poseen una EE del tipo [Sinic, Sproc, (Sres)], y (iii) la relación entre su significado en la variante de locado y su significado en la variante de locación es de oposición, pues se ven afectados por la incorporación a la matriz causativa $\mathrm{V}$ de las preposiciones que encabezan sus respectivos argumentos OBL.

b) Por lo que concierne a los VET en AP, nuestro análisis nos lleva a afirmar que poseen características opuestas a las anteriormente descritas: (i) son diádicos, (ii) como resultado de su carácter estativo presentan una EE definida exclusivamente por el subevento [Sinic], y (iii) su significado en la variante de locado es idéntico al que adquieren en la variante de locación, ya que los respectivos SP se circunscriben al ámbito del OD al que complementan sin afectar en modo alguno al significado verbal.

c) El hecho de que diversos tipos de verbos, además de los VET, posean la capacidad para combinarse con construcciones de AP permite, a nuestro juicio, confirmar la hipótesis de que esta clase de alternancia no es un subtipo de AL (de acuerdo con lo que, en oposición a Cifuentes 2008, 2010, sostiene Moreno Cabrera 2011), sino que constituye un fenómeno independiente y generalizado, capaz de afectar a múltiples y diversos tipos de verbos.

d) Por último, aunque previsiblemente diferencias aspectuales como las señaladas entre hacer, cometer y acabar y el mayor o menor grado de especificidad del significado verbal pueden incidir en el fenómeno, y sin perjuicio de lo que futuras investigaciones nos puedan aportar, nuestro análisis nos conduce a proponer dos requisitos mínimos para que un verbo pueda intervenir en la AP. Dichos requisitos están relacionados con nuestro 
conocimiento del mundo, es decir, con criterios pragmáticos, y son los siguientes: (i) que entre los referentes de locado y locación exista una relación de inclusión y que la predicación verbal abarque a ambos objetos.

\section{BIBLIOGRAFÍA}

CORPUS

ARIAS, G. (2015), «Enrique López presenta un escrito con más de 50 errores y faltas de ortografía» [en línea]. InfoLibre, 15 de septiembre de 2015 [Consulta: 28 de agosto de 2018]. Disponible en: <https://www.infolibre.es/noticias/politica/2015/09/15/el_ juez_enrique_lopez_presenta_escrito_con_mas_faltas_ortografia_quot_vallamos_ por_partes_quot_37746_1012.html >.

DIARIO ROTATIVO [redacción] (2016), «Julio Camejo muestra herida en el cuello tras ser asaltado» [en línea]. Querétaro, México, 19 de octubre de 2016 [Consulta: 1 de agosto de 2018]. Disponible en: <https://rotativo.com.mx/entretenimiento/569333julio-camejo-muestra-herida-cuello-tras-asaltado/>.

F. V. (2010), «¿Cómo explica las manchas de sangre en la camisa y el pantalón?» [en línea]. El Periódico de Aragón, 15 de septiembre de 2010 [consulta: 10 de octubre de 2018]. Disponible en: <http://www.elperiodicodearagon.com/noticias/aragon/com o-explica-manchas-sangre-camisa-pantalon_609850.html>.

FROST, M. (2017), «Consejos para mantener tu piel con buen aspecto» [en línea]. Muy fitness, 10 de mayo de 2017 [Consulta: 28 de agosto de 2018]. Disponible en: $<$ https://muyfitness.com/consejos-mantener-piel-lista_10980/>.

KOTIN, D. (2017), «La mujer joven con la lupa muestra la piel con acné» [en línea]. Dreamstime, 14 de junio de 2017 [Consulta: 30 de agosto de 2018]. Disponible en: $<$ https://es.dreamstime.com/la-mujer-joven-con-lupa-muestra-piel-acn\%C3\%A9image113029412>.

PATiÑO, F. (2017), «¡Oh, no! Primeras canas en la barba. ¿Qué puedes hacer?» [en línea]. Hombres de hoy Estilo y cuidado para nosotros, 12 de septiembre de 2017 [Consulta: 2 de agosto de 2018]. Disponible en: <http://www.mimundo.philips.es/oh-noprimeras-canas-en-la-barba-que-puedes-hacer/>.

PlAZA, V. (2015), «6 tips para lucir una corona de flores en el cabello» [en línea]. Matrimonios.cl, 12 de enero de 2015 [Consulta: 25 de agosto de 2018]. Disponible en: $<$ https://www.matrimonios.cl/articulos/6-tips-para-lucir-una-corona-deflores-en-el-cabello--c5740>.

\section{REFERENCIAS BIBLIOGRÁFICAS}

ANDERSON, S. (1971), «On the role of Deep Structure in semantic interpretation», Foundations of Language, 6, 197-219.

BAKER, M. (1997), «Thematic Roles and syntactic structure», en Elements of Grammar. Handbook of Generative Syntax, Haegeman, L. (ed.), Dordrechht, Kluver, 73-137.

BAKER, M. (1988), Incorporation. A Theory of Grammatical Function Changing, Chicago, The University of Chicago Press. 
BOSQUE, I. (1997), «Preposición tras preposición», en Contribuciones al estudio de la lingüistica hispánica. Homenaje al profesor Ramón Trujillo, Almeida, M. y Dorta, J. (eds.), Tenerife, Montesinos, 133-155.

BRINCKMAN, U. (1997), The locative alternation in German. Its structure and acquisition, Amsterdam-Filadelfia, John Benjamins.

Cifuentes HONRUBiA, J. L. (2008), «Removal verbs and locative alternations in Spanish», Estudios de Lingüística Universidad de Alicante, 22, 37-64.

Cifuentes HonrubiA, J. L. (2010), Clases semánticas y construcciones sintácticas: alternancias locativas en español, Lugo, Axac.

DOWTY, D. R. (1979), Word Meaning and Montague Grammar: The Semantics of Verbs and Times in Generative Semantics and in Montague's PTQ, Dordrecht, Reidel.

DOWTY, D. R. (2000), «The garden swarms with bees' and the fallacy of argument alternation», en Polysemy: Theoretical and Computational Approaches, Ravin, Y. y Leacock, C. (eds.), Oxford, Oxford University Press, 111-128.

DOWTY, D. R. (2003), «The dual analysis of adjuncts/complements in Categorial Grammar», en Modifying Adjuncts, Lang, E., Maienborn, C. y Fabricius-Hansen, C. (eds.), Berlín-Nueva York, Mouton de Gruyter, 33-66.

FILLMORE, C. J. (1968), «The case for the case», en Universals in Linguistic Theory, Bach, E. y Harms, R. T. (eds.), Nueva York, Holt, Rinehart y Winston, 1-88.

García Miguel, J. M. (2006), «Los complementos locativos», en Sintaxis histórica de la lengua española: la frase verbal, Company, C. (ed.), vol. 2, México, Universidad Nacional Autónoma de México, 1251-1336.

HALE, K. L. Y KEYSER, S. J. (1993), «On Argument Structure and the lexical expression of syntactic relations», en The View from Building 20: Essays in Linguistics in Honour of Sylvain Bromberger, Hale, K. L. y Keyser, S. J. (eds.), Cambridge, Massachusetts, MIT Press, 53-109.

HALE, K. L. Y KEYSER, SJ (1999), «Bound features, merge and transitivity alternations», MIT Working Papers in Linguistics, 35, 49-72.

HALL, B. (1965), Subject and Object in Modern English, tesis doctoral, Cambridge, MIT.

HARLEY, H. B. (2003), «Possession and the double object construction», Linguistic Variation Yearbook, 2, 29-68.

IWATA, S. (2005), «Locative alternation and two levels of verb meaning», Cognitive Linguistics, 16(2), 355-407.

IWATA, S. (2008), Locative Alternation: A lexical constructional approach, AmsterdamFiladelfia, John Benjamins.

JACKENDOFF, R. S. (1990), Semantic Structures, Cambridge, Massachusetts, MIT Press.

LARSON, R. K. (1990), «Double objects revisited: Reply to Jackendoff», Linguistic Inquiry, 21, 589-632.

LEVIN, B. (2003). Objecthood and object alternations [en línea]. Los Ángeles: Universidad de California (UCLA) [Consulta: 10 de agosto 2018]. Disponible en: $<$ https://web.stanford.edu/ bclevin/ucla03alt.pdf $>$.

LEVIN, B. (2006), English object alternations: A unified account [en línea]. California: Universidad de Stanford [Consulta: 7 de agosto de2018]. Disponible en: $<$ http://www.stanford.edu/bclevin/pubs.html>.

LEVIN, B. (2007), The Lexical semantics of verbs II: Aspectual approaches to Lexical Semantic Representation [en línea]. California: Universidad de Stanford, 1-3 de julio de 2007 
[Consulta: 20 de agosto de 2017]. Disponible en: $<$ https://web.stanford.edu/ bclevin/lsa07asp.pdf>.

LEVIN, B. y RAPPAPORT HOVAV, M. (1995), Unaccusativity at the Syntax-Lexical Semantics Interface, Cambridge, Massachusetts, MIT Press.

LEVIN, B. y RAPPAPORT HOVAV, M. (1998), «Morphology and Lexical Semantics», en The handbook of Morphology, Zwicky, A. y Spencer, A. (eds.), Oxford, Blackwell, 248271.

LEWANDOWSKI, W. (2014), La alternancia locativa en castellano y polaco: un análisis tipológico-construccional, tesis doctoral, Barcelona, Universidad Autónoma de Barcelona.

MACDONALD, J. E. (2006), The syntax of Inner Aspect, tesis doctoral, Stony, Brook University.

MAIENBORN, C. (2008), «On Davidsonian and Kimian states», en Existence: Semantics and Syntax, Comorovski, I. y Heusinger, K. (eds.), Dordrecht, Kluwer, 107-130.

MATEU, J. (2000), Lexicalization patterns and the Locative Alternation [en línea]. Bellaterra: Universidad Autónoma de Barcelona, 2000 [Consulta: 20 de mayo de 2018]. Disponible en: <http://filcat.uab.cat/clt/publicacions/reports/pdf/GGT-015.pdf $>$.

MATEU, J. (2002), Argument Structure: Relational construal of the syntax-semantics interface, tesis doctoral, Barcelona, Universidad Autónoma de Barcelona.

MATEU, J. (2017), «Two types of locative alternation», en Verb Valency Changes. Theoretical and typological perspectives. Typological Studies in Language 120, Álvarez, A. y Navarro, I. (eds.), Amsterdam-Filadelfia, John Benjamins.

MAYORAL HERNÁNDEZ, R. (2015), The Locative Alternation as an Unaccusative Construction: Verbs Types and Subject Position in Spanish, Múnich, Lincom GmbH.

MORENO CABRERA, J. C. (2011), «Las alternancias locativas y sus restricciones», en 60 problemas de gramática, Escandell M. V., Leonetti, M. y Sánchez López, C. (eds), Madrid, Ediciones Akal, 127-131.

NichOLs, J. (2008), «Prefixation and the Locative Alternation in Russian contact verbs», comunicación presentada en The Annual conference of the American Assosiation of Teachers of Slavic and East European Languages, San Francisco, 27-30 de diciembre de 2008.

PINKER, S. (1989), Learnability and Cognition: The Acquisition of Argument Structure, Cambridge, Massachusetts, MIT Press.

RAMCHAND, G. (2008), Verb meaning and the lexicon. Oxford, Oxford University Press.

RAPPAPORT HOVAV, M. y LEVIN, B. (1988), «What to do with theta-roles», en Syntax and Semantics 21: Thematic Relations, Wilkins, W. (ed.), San Diego, Academic Press, 736.

RothMAYR, A. (2009), The Structure of Stative Verbs, Amsterdam, John Benjamins.

STOWELL, T. (1981), Origins of Phrase Structure, tesis doctoral, Cambridge, MIT.

STOWELL, T. (1983), «Subjects across categories», The Linguistic Review, 2(3), 285-312. 